\title{
Summation of divergent power series by means of factorial series
}

\author{
Ernst Joachim Weniger \\ Institut für Physikalische und Theoretische Chemie, Universität Regensburg, D-93040 Regensburg, Germany
}

\begin{abstract}
Factorial series played a major role in Stirling's classic book Methodus Differentialis (1730), but now only a few specialists still use them. This article wants to show that this neglect is unjustified, and that factorial series are useful numerical tools for the summation of divergent (inverse) power series. This is documented by summing the divergent asymptotic expansion for the exponential integral $E_{1}(z)$ and the factorially divergent Rayleigh-Schrödinger perturbation expansion for the quartic anharmonic oscillator. Stirling numbers play a key role since they occur as coefficients in expansions of an inverse power in terms of inverse Pochhammer symbols and vice versa. It is shown that the relationships involving Stirling numbers are special cases of more general orthogonal and triangular transformations.
\end{abstract}

Keywords: Factorial series; Divergent asymptotic (inverse) power series; Stieltjes series; Quartic anharmonic oscillator; Stirling numbers; General orthogonal and triangular transformations;

2010 MSC: 11B73; 40A05; 40G99; 81Q15;

\section{Introduction}

Power series are extremely important analytical tools not only in mathematics, but also in the mathematical treatment of scientific and engineering problems. Unfortunately, a power series representation for a given function is from a numerical point of view a mixed blessing. A power series converges within its circle of convergence and diverges outside. Circles of convergence normally have finite radii, but there are many series expansions of considerable practical relevance, for example asymptotic expansions for special functions or quantum mechanical perturbation expansions, whose circles of convergence shrink to a single point.

The summation of divergent (inverse) power series is an old problem of mathematics, which is of considerable relevance also in related disciplines. Many different summation techniques have been developed which are often capable of associating a finite value to a divergent series. A highly condensed overview of various summation techniques was recently given in [54, Appendices A and B]. The role of summation techniques in theoretical physics was discussed in the recent review [17].

The topic of this article is the summation of divergent power series via so-called factorial series. A factorial series for a function $\Omega: \mathbb{C} \rightarrow \mathbb{C}$, which vanishes as $z \rightarrow+\infty$, is an expansion of the following type:

$$
\Omega(z)=\frac{a_{0}}{z}+\frac{a_{1} 1 !}{z(z+1)}+\frac{a_{2} 2 !}{z(z+1)(z+2)}+\cdots=\sum_{v=0}^{\infty} \frac{a_{v} v !}{(z)_{v+1}} .
$$

Here, $(z)_{v+1}=\Gamma(z+v+1) / \Gamma(z)=z(z+1) \ldots(z+v)$ is a Pochhammer symbol. The function $\Omega(z)$ represented by the factorial series (1.1) may have simple poles at $z=-m$ with $m \in \mathbb{N}_{0}$. The definition (1.1) is typical of the mathematical literature. It will become clear later that the separation of the series coefficients into a factorial $n$ ! and a reduced coefficient $a_{n}$ often offers formal advantages.

The use of factorial series for the summation of divergent (inverse) power series is not a new idea. There is Watson's classic article on the transformation of an asymptotic inverse power series to a convergent factorial series

Email address: joachim.weniger@chemie.uni-regensburg.de (Ernst Joachim Weniger) 
[45]. This topic was also considered in articles by Nevanlinna [30], Malgrange [28]. and Ramis and Thomann [36]. Thomann [42,43] discussed the summation of formal power series with the help of generalizations of factorial series, where the Pochhammer symbols $(z)_{n+1}=z(z+1) \ldots(z+n)$ are replaced by products $z\left(z+t_{1}\right) \ldots\left(z+t_{n}\right)$. There is also a recent article by Delabaere and Rasoamanana [20] on the connection of Borel summation and factorial series, which can be viewed to be an extension of a short discussion in Borel's classic book [6, pp. 234 - 245].

These references show that there is no lack of knowledge about factorial series in general and about their use as summation tools. Unfortunately, this knowledge is restricted to a relatively small group of specialists, and there is a deplorable lack of public awareness about factorial series and their numerical usefulness. My claim is supported by the fact that factorial series are not mentioned in the 2010 Mathematics Subject Classification (MSC), in the web database MathWorld (http: //mathworld. wolfram.com), or in Wikipedia (http://en.wikipedia.org/wiki/Main_Page). This neglect is not justified, and I believe that the full potential of factorial series as summation tools has not yet been realized.

Section 2 describes how I had become interested in factorial series. In Section 3 , the basic properties of factorial series are reviewed. In Section 4 it is shown that inverse power series and factorial series can be transformed into each other with the help of Stirling numbers. In Section 5, the transformation of Stieltjes series is considered. Section 6 describes the transformation of the divergent asymptotic series for the exponential integral to a convergent factorial series. In Section 7 a transformation for power series, which is essentially a factorial series in $1 / z$, is discussed, and in Section 8 , this transformation formula is used for the summation of the divergent Rayleigh-Schrödinger perturbation expansions for the ground state energy eigenvalue of the quartic anharmonic oscillator. This article is concluded by a short outlook in Section 9 Those properties of Stirling numbers, which are for our purposes most relevant, are reviewed in Appendix A In Appendix B, it is shown that the transformation formulas for inverse power and factorial series considered in Section 5 are just special cases of more general transformation formulas involving triangular and orthogonal matrices.

\section{My "rediscovery" of factorial series}

In 1985/1986 I became interested in Levin's sequence transformation [25], and I tried to understand the mathematical theory behind it. My interest was aroused by two articles by Smith and Ford [39, 40] who had shown that certain variants of Levin's sequence transformation were among the most powerful as well as most versatile sequence transformations known at that time.

In my work on Levin's sequence transformation [25] I discovered that its derivation becomes almost trivially simple if we start from the model sequence [46, Eq. (3.2-9)]

$$
s_{n}=s+\omega_{n} z_{n}, \quad n \in \mathbb{N}_{0} .
$$

The remainder estimates $\omega_{n}$ are assumed to be known, and the correction terms $z_{n}$ should be chosen in such a way that the products $\omega_{n} z_{n}$ provide sufficiently accurate and rapidly convergent approximations to the actual remainders $\left\{r_{n}\right\}_{n=0}^{\infty}$ of the sequence $\left\{s_{n}\right\}_{n=0}^{\infty}$ which is to be transformed.

In this approach, only the correction terms $\left\{z_{n}\right\}_{n=0}^{\infty}$ have to be determined. If good remainder estimates can be found, the determination of $z_{n}$ and the subsequent elimination of $\omega_{n} z_{n}$ from $s_{n}$ often leads to better results than the construction and subsequent elimination of other approximations to $r_{n}$.

The model sequence (2.1) has another indisputable advantage: A sequence transformation, which is exact for this model sequence, can be constructed easily under very mild conditions. Let us assume that a linear operator $\hat{T}$ can be found which annihilates the correction term $z_{n}$ for all $n \in \mathbb{N}_{0}$ according to $\hat{T}\left(z_{n}\right)=0$. Then we obtain a sequence transformation, which is exact for the model sequence (2.1), by applying $\hat{T}$ to the ratio $\left[s_{n}-s\right] / \omega_{n}=z_{n}$. Since $\hat{T}$ annihilates $z_{n}$ and is by assumption linear, the following sequence transformation $\mathscr{T}$ is exact for the model sequence (2.1) [46, Eq. (3.2-11)]:

$$
\mathscr{T}\left(s_{n}, \omega_{n}\right)=\frac{\hat{T}\left(s_{n} / \omega_{n}\right)}{\hat{T}\left(1 / \omega_{n}\right)}=s .
$$

The annihilation operator approach was introduced in [46, Section 3.2] in connection with my rederivation of Levin's transformation [25], but I also constructed in this way some other, closely related sequence transformations 
[46, Sections 7 - 9]. Brezinski and Redivo Zaglia [15, 16] and Brezinski and Matos [14] showed later that this approach is actually much more general: The majority of the currently known sequence transformations can be derived via (2.2) (for further references on this topic, see [52, p. 1214]).

We obtain a model sequence for Levin's sequence transformation [25] by assuming that $z_{n}$ in (2.1) is a truncated power series in $1 /(\beta+n)[46$, Eq. $(7.1-1)]$ :

$$
z_{n}=\sum_{j=0}^{k-1} \frac{c_{j}}{(\beta+n)^{j}}, \quad \beta>0 .
$$

The $k$ th power of the finite difference operator $\Delta$ defined by $\Delta f(n)=f(n+1)-f(n)$ annihilates an arbitrary polynomials $P_{k-1}(n)$ of degree $k-1$ in $n$. Thus, the weighted difference operator $\hat{T}=\Delta^{k}(\beta+n)^{k-1}$ is the appropriate annihilation operator for $z_{n}$ defined by [2.3], and Levin's sequence transformation [25] can in the notation of [46, Eqs. (7.1-6) and (7.1-7)] be expressed as follows:

$$
\mathscr{L}_{k}^{(n)}\left(\beta, s_{n}, \omega_{n}\right)=\frac{\Delta^{k}\left[(\beta+n)^{k-1} s_{n} / \omega_{n}\right]}{\Delta^{k}\left[(\beta+n)^{k-1} / \omega_{n}\right]}=\frac{\sum_{j=0}^{k}(-1)^{j}\left(\begin{array}{c}
k \\
j
\end{array}\right) \frac{(\beta+n+j)^{k-1}}{(\beta+n+k)^{k-1}} \frac{s_{n+j}}{\omega_{n+j}}}{\sum_{j=0}^{k}(-1)^{j}\left(\begin{array}{c}
k \\
j
\end{array}\right) \frac{(\beta+n+j)^{k-1}}{(\beta+n+k)^{k-1}} \frac{1}{\omega_{n+j}}}, \quad k, n \in \mathbb{N}_{0} .
$$

The numerator and denominator sums of this and of related transformations can also be computed recursively [52, Section III].

This undeniable success inspired me to look for other applications of the annihilation operator approach. If we replace in (2.3) the powers $(\beta+n)^{j}$ by Pochhammer symbols $(\beta+n)_{j}$, we obtain a truncated factorial series in $\beta+n$ :

$$
z_{n}=\sum_{j=0}^{k-1} \frac{c_{j}^{\prime}}{(\beta+n)_{j}}, \quad \beta>0 .
$$

Now, $\hat{T}=\Delta^{k}(\beta+n)_{k-1}$ is the appropriate annihilation operator, and we obtain [46, Eqs. (8.2-6) and (8.2-7)]:

$$
\mathscr{S}_{k}^{(n)}\left(\beta, s_{n}, \omega_{n}\right)=\frac{\Delta^{k}\left[(\beta+n)_{k-1} s_{n} / \omega_{n}\right]}{\Delta^{k}\left[(\beta+n)_{k-1} / \omega_{n}\right]}=\frac{\sum_{j=0}^{k}(-1)^{j}\left(\begin{array}{c}
k \\
j
\end{array}\right) \frac{(\beta+n+j)_{k-1}}{(\beta+n+k)_{k-1}} \frac{s_{n+j}}{\omega_{n+j}}}{\sum_{j=0}^{k}(-1)^{j}\left(\begin{array}{c}
k \\
j
\end{array}\right) \frac{(\beta+n+j)_{k-1}}{(\beta+n+k)_{k-1}} \frac{1}{\omega_{n+j}}}, \quad k, n \in \mathbb{N}_{0} .
$$

My derivation of this sequence transformations, whose theory was developed in [46, Section 8], was entirely based on heuristics. I had only looked for situations in which I could apply the annihilation operator formalism (2.2) effectively. If the correction term $z_{n}$ is according to (2.5) a truncated factorial series, it can be annihilated easily, but I had no idea whether the resulting sequence transformation $\mathscr{S}_{k}^{(n)}\left(\beta, s_{n}, \omega_{n}\right)$ would be computationally useful or not.

$\mathscr{S}_{l}^{(n)}\left(\beta, s_{n}, \omega_{n}\right)$ was first used for the evaluation of auxiliary functions in molecular electronic structure calculations [56]. Later, it was used with considerable success in the case of slowly convergent or divergent alternating series (numerous references are listed in [52, p. 1225]). Currently, $\mathscr{S}_{k}^{(n)}\left(\beta, s_{n}, \omega_{n}\right)$ is used quite a lot in optics [7-13, 26, 27].

When I constructed $\mathscr{S}_{k}^{(n)}\left(\beta, s_{n}, \omega_{n}\right)$ in 1986, I had no idea what the correction term 2.5) actually is: Factorial series had not been part of my mathematical training. It took a while until I found out that 2.5) is a truncated factorial series, and that Nielsen's classic book [31], which is still one of the principal references on factorial series, had already been waiting for quite a while on my bookshelf.

In my later work on convergence acceleration and summation processes, I noticed that $\mathscr{L}_{k}^{(n)}\left(\beta, s_{n}, \omega_{n}\right)$ and $\mathscr{S}_{k}^{(n)}\left(\beta, s_{n}, \omega_{n}\right)$ usually have similar, but not identical properties. Nevertheless, in some cases spectacular differences were observed. For example, in summation calculations for the divergent Rayleigh-Schrödinger perturbation expansions of the ground state energies of anharmonic oscillators [55], we observed that Levin's transformation 
$\mathscr{L}_{k}^{(n)}\left(\beta, s_{n}, \omega_{n}\right)$ ultimately produces divergent results, whereas $\mathscr{S}_{k}^{(n)}\left(\beta, s_{n}, \omega_{n}\right)$ produces very good results (see also [48]). A similar divergence of Levin's transformation was also observed by Č́ížek, Zamastil, and Skála [19]. Needless to say that these observations puzzled me.

The construction of Levin's transformation is based on the assumption that the ratio $\left[s_{n}-s\right] / \omega_{n}$ can be expressed as an inverse power series, whereas $\mathscr{S}_{k}^{(n)}\left(\beta, s_{n}, \omega_{n}\right)$ implicitly assumes that $\left[s_{n}-s\right] / \omega_{n}$ can be expressed as a factorial series. For me, it was a plausible hypothesis that the observed differences of $\mathscr{L}_{k}^{(n)}\left(\beta, s_{n}, \omega_{n}\right)$ and $\mathscr{S}_{k}^{(n)}\left(\beta, s_{n}, \omega_{n}\right)$ could be related to different properties of inverse power and factorial series, respectively. Because of my complete lack of knowledge about factorial series, I first had to study their properties. The results presented in this article are in some sense a by-product of these studies.

\section{Basic properties of factorial series}

Factorial series have been known for a very long time. In Tweedle's annotated translations of Stirling's classic Methodus Differentialis it is remarked that Stirling was not the inventor of factorial series. Apparently, Stirling became aware of factorial series by the work of the French mathematician Nicole [44, p. 174]. However, Stirling used factorial series extensively and thus did a lot to popularize them.

The application of higher powers of the finite difference operator $\Delta=\Delta_{z}$ to a factorial series in $z$ yields an extremely compact result. If we use $\Delta_{z}^{k}\left[n ! /(z)_{n+1}\right]=(-1)^{k}(n+k) ! /(z)_{n+k+1}$ with $k \in \mathbb{N}_{0}$, we obtain

$$
\Delta_{z}^{k} \Omega(z)=\sum_{v=0}^{\infty} \Delta_{z}^{k} \frac{a_{v} v !}{(z)_{v+1}}=(-1)^{k} \sum_{\nu=0}^{\infty} \frac{a_{v}(v+k) !}{(z)_{v+k+1}}=(-1)^{k} \sum_{\kappa=k}^{\infty} \frac{a_{\kappa-k} \kappa !}{(z)_{\kappa+1}} .
$$

Factorial series play a similar role in the theory of difference equations as inverse power series in the theory of differential equations, and classic books on finite difference such as the ones by Milne-Thomson [29], or Nörlund [32-34]) treat factorial series. A contemporary discussion of the use of factorial series in the context of difference equations can be found in a recent article by Olde Daalhuis [35].

But I am are much more interested in the convergence properties of factorial series, which fortunately can be analyzed easily. If we use [1, Eq. (6.1.47) on p. 257]

$$
\Gamma(z+a) / \Gamma(z+b)=z^{a-b}[1+\mathrm{O}(1 / z)], \quad z \rightarrow \infty
$$

we obtain the asymptotic estimate $n ! /(z)_{n+1}=\mathrm{O}\left(n^{-z}\right)$ as $n \rightarrow \infty$. Thus, the factorial series (1.1) converges with the possible exception of the points $z=-m$ with $m \in \mathbb{N}_{0}$ if and only if the associated Dirichlet series $\tilde{\Omega}(z)=\sum_{n=1}^{\infty} a_{n} / n^{z}$ converges (see for example [22, p. 262] or [23, p. 167]). Accordingly, a factorial series converges for sufficiently large $\mathfrak{R}(z)$ even if the reduced series coefficients $a_{n}$ in 1.1 grow like a fixed power $n^{\alpha}$ with $\alpha>0$ as $n \rightarrow \infty$.

Factorial series are closely related to the beta function, which is usually defined as the following ratio of gamma functions [1, Eq. (6.2.2)]:

$$
B(x, y)=\frac{\Gamma(x) \Gamma(y)}{\Gamma(x+y)}, \quad x, y \in \mathbb{C} .
$$

Thus, the ratio $n ! /(z)_{n+1}$ can be expressed as a beta function:

$$
B(z, n+1)=\frac{n !}{(z)_{n+1}} .
$$

Accordingly, a factorial series can also be expressed as an expansion in terms of beta functions [29, p. 288]:

$$
\Omega(z)=\sum_{n=0}^{\infty} a_{n} B(z, n+1) .
$$

The beta function possesses numerous integral representations. For our purposes the most useful one is the socalled Euler integral of the first kind (see for example [1, Eq. (6.2.1)]):

$$
B(x, y)=\int_{0}^{1} t^{x-1}(1-t)^{y-1} \mathrm{~d} t, \quad \Re(x), \Re(y)>0 .
$$


Combination of (3.5) and (3.6) yields the following integral representation:

$$
\frac{n !}{(z)_{n+1}}=\int_{0}^{1} t^{z-1}(1-t)^{n} \mathrm{~d} t, \quad \mathfrak{R}(z)>0 \quad n \in \mathbb{N}_{0} .
$$

If we now combine (3.4), (3.5) and (3.7) and interchange integration and summation, we obtain the following integral representation (see for instance [31, Satz I on p. 244] or [29, p. 289]):

$$
\begin{aligned}
\Omega(z) & =\int_{0}^{1} t^{z-1} \varphi(t) \mathrm{d} t, \quad \Re(z)>0, \\
\varphi(t) & =\sum_{n=0}^{\infty} a_{n}(1-t)^{n} .
\end{aligned}
$$

This integral representation is of considerable importance: Frequently, the properties of $\Omega(z)$ can be studied more conveniently via the corresponding properties of the conjugated function $\varphi(t)$ than via the defining factorial series (1.1) (see for example [31, Kapitel XVII]). As discussed in Section 8, this integral representation can also be used for the evaluation of factorial series.

\section{Transformations of inverse power series and factorial series}

Inverse powers $1 / z^{k+1}$ and inverse Pochhammer symbols $1 /(z)_{k+1}$ can be transformed into each other via (A.11) and (A.14), respectively. Therefore, inverse power series and factorial series can also be transformed into each other. This has been known for a very long time. The algebraic processes effecting these transformations were already described in Nielsen's book [31] which was first published in 1906. It seems, however, that these potentially very useful transformation formulas are now largely forgotten.

Let us assume that a function $\psi: \mathbb{C} \rightarrow \mathbb{C}$ possesses the inverse power series $\psi(z)=\sum_{n=0}^{\infty} c_{n} / z^{n+1}$. If we insert A.14 into this series and rearrange the order of summations, we obtain the transformation formula

$$
\sum_{n=0}^{\infty} \frac{c_{n}}{z^{n+1}}=\sum_{m=0}^{\infty} \frac{(-1)^{m}}{(z)_{m+1}} \sum_{\mu=0}^{m}(-1)^{\mu} \mathbf{S}^{(1)}(m, \mu) c_{\mu},
$$

which shows that the coefficients of the factorial series are weighted averages of the power series coefficients involving Stirling numbers of the first kind. I employed this transformation already in [53] in order to speed up the convergence of truncated asymptotic expansions for the truncation errors of series expansions for special functions.

An inverse expansion can also be derived. Let us assume that a function $\chi: \mathbb{C} \rightarrow \mathbb{C}$ possesses a factorial series $\chi(z)=\sum_{n=0}^{\infty} d_{n} /(z)_{n+1}$. If we insert A.11 into this series and rearrange the order of summations, we obtain the transformation formula

$$
\sum_{n=0}^{\infty} \frac{d_{n}}{(z)_{n+1}}=\sum_{m=0}^{\infty} \frac{(-1)^{m}}{z^{m+1}} \sum_{\mu=0}^{m}(-1)^{\mu} \mathbf{S}^{(2)}(m, \mu) d_{\mu} .
$$

The operations producing the transformation formulas (4.1) and (4.2) are purely formal. Therefore, we cannot tacitly assume that the inverse power or the factorial series necessarily converge. This has to be checked explicitly in each case.

The sign patterns in the inner sums $\sum_{\mu=0}^{m}(-1)^{\mu} \mathbf{S}^{(1)}(m, \mu) c_{\mu}$ and $\sum_{\mu=0}^{m}(-1)^{\mu} \mathbf{S}^{(2)}(m, \mu) d_{\mu}$ in (4.1) and (4.2) are of crucial importance for the convergence or divergence of the formal expansions (4.1) and (4.2). If the signs of the terms in the inner sums alternate, we can hope for a substantial cancellation as in binomial sums $\sum_{j=0}^{k}(-1)^{k}\left(\begin{array}{c}k \\ j\end{array}\right) f_{n+j}$, but if all terms have the same sign, a potentially explosive accumulation can take place. In the former case, convergence is likely, while in the latter case we should be prepared for divergence.

The ability of the Stirling numbers of the first kind to achieve a cancellation is immediately obvious from its finite generating function. Setting $z=k$ with $1 \leq k \leq n-1$ in A.1 yields $\sum_{v=0}^{n} k^{v} \mathbf{S}^{(1)}(n, v)=0$ for $n \geq 2$. Let us now assume that the coefficients $c_{n}$ in (4.1) have strictly alternating signs. Consequently, A.3 implies that the terms of the inner sum $(-1)^{m} \sum_{\mu=0}^{m}(-1)^{\mu} \mathbf{S}^{(1)}(m, \mu) c_{\mu}=\sum_{\mu=0}^{m}\left|\mathbf{S}^{(1)}(m, \mu)\right| c_{\mu}$ in (4.1) also have strictly alternating signs, and we can hope for a substantial cancellation. The inner sum should for large values of the outer index $m$ be much smaller in 
magnitude than its individual terms. Because of this cancellation, we can hope that a function defined by a divergent inverse power series with strictly alternating coefficients can be expressed and computed by a convergent factorial series.

The situation is not nearly as nice if the coefficients $c_{n}$ in (4.1) all have the same sign. If we set in (A.2) $z=$ 1 and use A.3), we obtain $(-1)^{m} \sum_{\mu=0}^{m}(-1)^{\mu} \mathbf{S}^{(1)}(m, \mu)=\sum_{\mu=0}^{m}\left|\mathbf{S}^{(1)}(m, \mu)\right|=m$ !. Accordingly, the coefficients $\sum_{\mu=0}^{m}\left|\mathbf{S}^{(1)}(m, \mu)\right| c_{\mu}$ of the factorial series should be (much) larger than the coefficients $c_{n}$ of the inverse power series.

\section{Stieltjes series}

A function $F: \mathbb{C} \rightarrow \mathbb{C}$ is called a Stieltjes function if it can be expressed by the Stieltjes integral

$$
F(z)=\int_{0}^{\infty} \frac{\mathrm{d} \Phi(t)}{z+t}, \quad|\arg (z)|<\pi .
$$

Here, $\Phi(t)$ is a bounded, nondecreasing function taking infinitely many different values on the interval $0 \leq t<\infty$. Moreover, the moment integrals

$$
\mu_{n}=\int_{0}^{\infty} t^{n} \mathrm{~d} \Phi(t), \quad n \in \mathbb{N}_{0},
$$

must be positive and finite for all finite values of $n$.

Detailed discussions of Stieltjes series and their special role in the theory of summability can be found in the books by Bender and Orszag [3, Chapter 8.6] or Baker and Graves-Morris [2, Chapter 5]. In the case of divergent Stieltjes series, it can be shown rigorously that the Padé approximants $[n+j / n]$ with fixed $j \geq-1$ converge to a uniquely determined Stieltjes function as $n \rightarrow \infty$.

An inverse power series representation for such a Stieltjes function $F(z)$ can be derived easily. We insert the geometric series $\sum_{v=0}^{\infty}(-t)^{v} / z^{v+1}=1 /(z+t)$, which converges for $|t / z|<1$, into the integral representation (5.1) and - ignoring all questions of legitimacy and convergence - integrate term-wise from 0 to $\infty$ using (5.2). Thus, a Stieltjes function $F(z)$ can at least formally be represented by its Stieltjes series

$$
F(z)=\sum_{v=0}^{\infty} \frac{(-1)^{v} \mu_{v}}{z^{v+1}}
$$

A factorial series for a a Stieltjes function $F(z)$ can also be derived quite easily. For that purpose, we use the convergent factorial series (A.12) with $w=-t$ in the integral representation (5.1) and interchange integration and summation:

$$
\begin{aligned}
F(z) & =\int_{0}^{\infty} \sum_{n=0}^{\infty} \frac{(-t)_{n}}{(z)_{n+1}} \mathrm{~d} \Phi(t) \\
& =\sum_{n=0}^{\infty} \frac{1}{(z)_{n+1}} \int_{0}^{\infty}(-t)_{n} \mathrm{~d} \Phi(t) .
\end{aligned}
$$

If we now expand $(-t)_{n}$ via A.1) and do the resulting moment integrals according to (5.2), we obtain:

$$
\begin{aligned}
F(z) & =\sum_{n=0}^{\infty} \frac{(-1)^{n}}{(z)_{n+1}} \sum_{v=0}^{n} \mathbf{S}^{(1)}(n, v) \int_{0}^{\infty} t^{v} \mathrm{~d} \Phi(t) \\
& =\sum_{n=0}^{\infty} \frac{(-1)^{n}}{(z)_{n+1}} \sum_{v=0}^{n} \mathbf{S}^{(1)}(n, v) \mu_{v}
\end{aligned}
$$

Obviously, the factorial series (5.7) for a Stieltjes function is a special case of the more general result (4.1).

Again, cancellation is the reason why we can hope that the factorial series 5.7 converges and is computationally useful even if the corresponding Stieltjes series (5.3) diverges. The power $t^{n}$ in the moment integral (5.2) is positive, but the Pochhammer symbol $(-t)_{n}$ in the generalized moment integrals $\int_{0}^{\infty}(-t)_{n} \mathrm{~d} \Phi(t)$ in $(5.5)$ has zeros for $t=$ $0,1, \ldots n-1$ and alternates in sign. This leads to a substantial cancellation. 


\section{Summation of the asymptotic series for the exponential integral}

The practical usefulness of the transformation of a factorially divergent inverse power series to a convergent factorial series via (4.1) or via (5.7) in the case of a Stieltjes series can be demonstrated by means of the exponential integral [1, Eq. (5.1.1)]

$$
E_{1}(z)=\int_{z}^{\infty} \frac{\exp (-t) \mathrm{d} t}{t}
$$

which possesses the following asymptotic expansion as $z \rightarrow \infty$ [1, Eq. (5.1.51)],

$$
z \mathrm{e}^{z} E_{1}(z) \sim \sum_{m=0}^{\infty} \frac{(-1)^{m} m !}{z^{m}}={ }_{2} F_{0}(1,1 ;-1 / z), \quad z \rightarrow \infty .
$$

The exponential integral can also be expressed as a Stieltjes integral [1, Eq. (5.1.28)]:

$$
\mathrm{e}^{z} E_{1}(z)=\int_{0}^{\infty} \frac{\exp (-t) \mathrm{d} t}{z+t}=\frac{1}{z} \int_{0}^{\infty} \frac{\exp (-t) \mathrm{d} t}{1+t / z}
$$

Thus, the divergent inverse power series (6.2) is a Stieltjes series.

If we now combine (6.2) with (5.6) and (5.7), we obtain:

$$
\mathrm{e}^{z} E_{1}(z)=\sum_{n=0}^{\infty} \frac{1}{(z)_{n+1}} \int_{0}^{\infty}(-t)_{n} \mathrm{e}^{-t} \mathrm{~d} t=\sum_{n=0}^{\infty} \frac{(-1)^{n}}{(z)_{n+1}} \sum_{v=0}^{n} \mathbf{S}^{(1)}(n, v) v !
$$

As discussed in Sections 4 and 5 the transformation of an inverse power series to a factorial series only produces a numerically useful result if a substantial cancellation takes place in the inner sums in (4.1) or (5.7). For that purpose, the inverse power series coefficients $(-1)^{n} n$ ! and the factorial series coefficients $(-1)^{n} \sum_{v=0}^{n} \mathbf{S}^{(1)}(n, v) v$ ! are displayed in Table 1

Table 1: Leading coefficients of the inverse power and the factorial series of the exponential integral.

\begin{tabular}{rrr}
\hline \hline$n$ & $(-1)^{n} n !$ & $(-1)^{n} \sum_{v=0}^{n} \mathbf{S}^{(1)}(n, v) v !$ \\
\hline 0 & 1 & 1 \\
1 & -1 & -1 \\
2 & 2 & 1 \\
3 & -6 & -2 \\
4 & 24 & 4 \\
5 & -120 & -14 \\
6 & 720 & 38 \\
7 & -5040 & -216 \\
8 & 40320 & 600 \\
9 & -362880 & -6240 \\
10 & 3628800 & 9552 \\
11 & -39916800 & -319296 \\
12 & 479001600 & -519312 \\
13 & -6227020800 & -28108560 \\
14 & 87178291200 & -176474352 \\
\hline \hline
\end{tabular}

The numbers displayed in Table 1 show that there is indeed a substantial amount of cancellation in the inner sums in (6.4): The coefficients of the inverse power series grow much more rapidly in magnitude than the coefficients of the 
factorial series. This is enough to produce a convergent result. For example, the first 15 coefficients of the factorial series produce for $z=5$ the following result:

$$
\frac{\sum_{n=0}^{14} \frac{(-1)^{n}}{(5)_{n+1}} \sum_{v=0}^{n} \mathbf{S}^{(1)}(n, v) v !}{\exp (5) E_{1}(5)}=1.000000764
$$

\section{Conversion of a power series to a factorial series}

Assume that a function $f: \mathbb{C} \rightarrow \mathbb{C}$ possesses a power series $f(z)=\sum_{n=0}^{\infty} \gamma_{n} z^{n}$. For a transformation of this power series to a factorial series, we express it as an inverse power series in $1 / z$ :

$$
f(z)=\frac{1}{z} \sum_{n=0}^{\infty} \frac{\gamma_{n}}{(1 / z)^{n+1}}
$$

If we now use (4.1), we obtain a factorial series in $1 / z$ :

$$
\sum_{n=0}^{\infty} \gamma_{n} z^{n}=\frac{1}{z} \sum_{m=0}^{\infty} \frac{(-1)^{m}}{(1 / z)_{m+1}} \sum_{\mu=0}^{m}(-1)^{\mu} \mathbf{S}^{(1)}(m, \mu) \gamma_{\mu}
$$

An equivalent factorial series was considered by Ramis and Thomann [36, p. 20]. Thomann ([42, p. 526] and [43, Section 5.3]) considered similar expansions in terms of generalized factorial series, where the Pochhammer symbols are replaced by products $z\left(z+t_{1}\right) \ldots\left(z+t_{n}\right)$. It is clear that generalized factorial series are at least potentially more powerful than their ordinary counterparts. However, it is not a priori clear how the parameters $\left\{t_{n}\right\}_{n=1}^{\infty}$ should be chosen. In addition, Thomann's formulas contain so-called generalized Stirling numbers instead of the ordinary Stirling numbers of the first kind. Therefore, it is not immediately obvious whether Thomann's generalized transformation formula is really more useful than (7.2).

Further manipulations of the Pochhammer symbol in (7.2) are possible:

$$
\frac{1}{(1 / z)_{m+1}}=\frac{1}{\prod_{k=0}^{m}[k+1 / z]}=\frac{z^{m+1}}{\prod_{k=1}^{m} k[z+1 / k]}=\frac{z}{m !} \prod_{k=1}^{m} \frac{z}{z+1 / k} .
$$

Inserting this into 7.2 yields:

$$
\sum_{v=0}^{\infty} \gamma_{v} z^{v}=\sum_{m=0}^{\infty} \frac{(-1)^{m}}{m !} \prod_{k=1}^{m} \frac{z}{z+1 / k} \sum_{\mu=0}^{m}(-1)^{\mu} \mathbf{S}^{(1)}(m, \mu) \gamma_{\mu} .
$$

If the power series coefficients $\gamma_{v}$ have strictly alternating signs, we can expect cancellation in the inner sum involving the Stirling numbers. Moreover, for $z>0$ we have $z /(z+1 / k)<1$ for $k \in \mathbb{N}$. Since the transformation (7.3) also produces the factorial $1 / m$ !, we can expect that the transformation 7.4 produces a convergent and numerically useful result even if the coefficients $\gamma_{v}$ diverge factorially in magnitude.

\section{The quartic anharmonic oscillator}

In their seminal articles [4, 5], Bender and Wu showed that the Rayleigh-Schrödinger perturbation expansions for the energy eigenvalues $E^{(m)}(\beta)$ of the anharmonic oscillators defined by the Hamiltonians

$$
\hat{H}^{(m)}(\beta)=\hat{p}^{2}+\hat{x}^{2}+\beta \hat{x}^{2 m}, \quad m=2,3,4, \ldots, \quad \hat{p}=-\mathrm{i} \frac{\mathrm{d}}{\mathrm{d} x}
$$

diverge quite violently for every nonzero coupling constant $\beta$ (here, the same notation as in [50, 51, 55] is used). Later, perturbation expansions with a similar type of divergence were discovered in the case of other quantum mechanical 
systems, which ultimately created a new sub-discipline of theoretical physics called large order perturbation theory (see for example the book by Le Guillou and Zinn-Justin [24] and the articles reprinted there).

In the following years, a lot of work has been done on the summation of divergent perturbation expansions (a mathematically oriented overview can be found in [38]). In particular the quartic anharmonic oscillator with $m=2$ in (8.1) has become a very popular computational laboratory for theoretical physicists. In spite of its simplicity, the quartic anharmonic oscillator leads to challenging computational and conceptual problems, as documented in countless articles (far too many to be cited here).

In this article, I am exclusively interested in the summation of the divergent Rayleigh-Schrödinger perturbation expansion

$$
E^{(2)}(\beta)=\sum_{n=0}^{\infty} b_{n}^{(2)} \beta^{n}
$$

for the ground state energy of the quartic anharmonic oscillator by transforming it to a factorial series via (7.4). Long, but nevertheless incomplete lists of references dealing with other approaches for the summation of the divergent perturbation expansions of the anharmonic oscillators can be found in [50, 51, 55] or also in [49, Kap. 10].

If the convention 8.1 for the Hamiltonian is used, the coefficients $b_{n}^{(2)}$ possess in the case of large indices $n$ the following leading order asymptotics (see for example [50, Eq. (2.3)]):

$$
b_{n}^{(2)} \sim(-1)^{n+1} \frac{(24)^{1 / 2}}{\pi^{3 / 2}} \Gamma(n+1 / 2)(3 / 2)^{n}, \quad n \rightarrow \infty .
$$

This asymptotic estimate shows that the perturbation series $(8.2)$ diverges for all $\beta \neq 0$ like the generalized hypergeometric series ${ }_{2} F_{0}(1 / 2,1 ;-3 \beta / 2)=\sum_{m=0}^{\infty}(1 / 2)_{m}(-3 \beta / 2)^{m}$ [47, Eq. (1.10)].

It was shown rigorously bu Simon [37, Theorem IV.2.1] that the perturbation expansion

$$
\Delta E^{(2)}(\beta)=\sum_{n=0}^{\infty} b_{n+1}^{(2)} \beta^{n}
$$

for the energy shift defined by $E^{(2)}(\beta)=b_{0}^{(2)}+\beta \Delta E^{(2)}(\beta)=1+\beta \Delta E^{(2)}(\beta)$ is a Stieltjes series. This is a highly advantageous feature. As discussed in Section 5, this implies that the perturbation series (8.4) corresponds to a uniquely defined Stieltjes function since it is Padé summable. Moreover, the asymptotic estimate (8.3) implies that the terms of the perturbation expansion (8.4) have for $\beta>0$ strictly alternating signs (see also [49, Tabelle 10-1]), which is advantageous if we want to sum it with the help of (7.4).

If we transform the perturbation series (8.4) for the energy shift with the help of (7.4), we obtain the following expansion for the ground state energy of the quartic oscillator:

$$
E^{(2)}(\beta)=1+\beta \sum_{m=0}^{\infty} \frac{(-1)^{m}}{m !} \prod_{k=1}^{m} \frac{\beta}{\beta+1 / k} \sum_{\mu=0}^{m}(-1)^{\mu} \mathbf{S}^{(1)}(m, \mu) b_{\mu+1}^{(2)} .
$$

The first 34 terms of the infinite series on the right-hand side yield for $\beta=1 / 5$ the energy $E_{\mathrm{FS}}^{(2)}(1 / 5)=1.118305 \ldots$, which is less accurate than the energy $E_{\mathrm{PA}}^{(2)}(1 / 5)=1.118292654373 \ldots$ obtained by computing the Padé approximants [17/17] from the first 34 terms of the perturbation expansion (8.4) for the energy shift $\Delta E^{(2)}(1 / 5)$. These approximations can be compared to the "exact" energy $E_{\text {exact }}^{(2)}(1 / 5)=1.118292654367039154 \ldots$ obtained by a very sophisticated summation calculation [49, Tabelle 10-9].

Thus, the truncated series expansion (8.5), which does a linear transformation of the perturbation series coefficients $b_{1}, b_{2}, \ldots, b_{34}$, is less efficient than the highly nonlinear Padé approximant using the same number of coefficients $b_{\mu}^{(2)}$. Nevertheless, improvements are possible if we use the integral representation (3.8) for the evaluation of the corresponding factorial series $\Omega(z)=\sum_{n=0}^{\infty} a_{n} n ! /(z)_{n+1}$.

A direct use of a truncation of the power series $\varphi(t)=\sum_{n=0}^{\infty} a_{n}(1-t)^{n}$ defined by $(3.8 \mathrm{~b}$ ) in the integral representation 3.8a does not lead to an improvement since integration is linear. It is, however, possible to replace the truncated power series for $\varphi(t)$ by a Padé approximant in $1-t$ and to evaluate the resulting expression by numerical quadrature. The use of the Padé approximant [17/17] to $\varphi(t)$ in $(3.8)$ yields $E_{\operatorname{IntFS}}(1 / 5)=1.118292654369 \ldots$, 
which is better than the direct Padé summation result $E_{\mathrm{PA}}^{(2)}(1 / 5)=1.118292654373 \ldots$, but less accurate than $E_{\mathrm{BP}}(1 / 5)=1.118292654367039152 \ldots$ obtained by doing a so-called Borel-Padé transformation originally introduced in [21].

\section{Outlook}

A single article cannot provide an exhaustive treatment of the numerical utilization of factorial series for the summation of divergent (inverse) power series. Because of space limitations, many interesting or potentially useful aspect of the theory of factorial series were treated only superficially or even completely ignored. Nevertheless, I hope that this article will inspire others.

Inner sums of the type of $(-1)^{m} \sum_{\mu=0}^{m}(-1)^{\mu} \mathbf{S}^{(1)}(m, \mu) c_{\mu}$ occurring on the right-hand side of (4.1) are the key quantities of this article. Recurrence formulas or even alternative closed form expressions would obviously be desirable. In the case of Stieltjes series, a recursive scheme for the computation of the generalized moment integrals $\int_{0}^{\infty}(-t)_{n} \mathrm{~d} \Phi(t)$ in 5.5 can be derived.

The numerical examples of this article also raise questions. It is an obvious question whether and how well the convergence of factorial series can be accelerated by sequence transformations. When I looked at the convergence of the factorial series (6.4) for $E_{1}(z)$, I applied sequence transformations to speed up its convergence. However, convergence was not improved substantially by the transformations I used. At the moment, it is unclear whether this is a specific feature of the factorial series (6.4), or whether we face a problem of a more general nature. It could be that the convergence of factorial series can only be accelerated effectively if other, specially designed sequence transformations are used. This should be investigated.

It should be worthwhile to investigate whether the transformation formula (7.4) can also be used profitably in the case of convergent power series as a convergence acceleration tool. One can also hope that in the case of sufficiently simple power series coefficients $\gamma_{n}$ explicit expressions for the inner sum $(-1)^{m} \sum_{\mu=0}^{m}(-1)^{\mu} \mathbf{S}^{(1)}(m, \mu) \gamma_{\mu}$ can be found, which would yield new explicit expressions in terms of factorial series for functions defined by power series.

In Section 8 , the truncated power series $\varphi(t)=\sum_{n=0}^{\infty} a_{n}(1-t)^{n}$ defined by $(3.8 \mathrm{~b})$ was converted to a Padé approximant in $1-t$, which was inserted into the integral representation (3.8). One should investigate whether other sequence transformations produce better approximations to $\varphi(t)$ than Padé approximants.

\section{Appendix A. Stirling numbers}

With respect to notation, the theory of Stirling numbers is a mess. This is partly due to the fact that numerous different symbols are used in the literature (a discussion of the various notations can be found in [1, p. 822]). To make things worse, different and incompatible notations are used for factorial expressions. In special function theory, Pochhammer symbols $(z)_{n}=z(z+1) \ldots(z+n-1)=\Gamma(z+n) / \Gamma(z)$ are consistently used, but in combinatorics, it is more common to use instead falling factorials $z(z-1) \ldots(z-n+1)=\Gamma(z+1) / \Gamma(z-n+1)$. Unfortunately, in the literature of combinatorics falling factorials are often denoted by the symbol $(z)_{n}$ normally reserved in special function theory for Pochhammer symbols.

The Stirling numbers $\mathbf{S}^{(1)}(n, v)$ of the first kind are the polynomial coefficients of a Pochhammer symbol $(z-n+$ $1)_{n}=z(z-1) \ldots(z-n+1)=\Gamma(z+1) / \Gamma(z-n+1)$ (see for example [41, Eq. (1) on p. 56]):

$$
(z-n+1)_{n}=(-1)^{n}(-z)_{n}=\sum_{v=0}^{n} \mathbf{S}^{(1)}(n, v) z^{v}, \quad n \in \mathbb{N}_{0}
$$

If we use $(z-n+1)_{n}=(-1)^{n}(-z)_{n}$ and replace $z$ by $-z$, we obtain:

$$
(z)_{n}=(-1)^{n} \sum_{v=0}^{n}(-1)^{v} \mathbf{S}^{(1)}(n, v) z^{v}, \quad n \in \mathbb{N}_{0}
$$

If $z>0$ holds, the coefficients of all powers $z^{v}$ with $0 \leq v \leq n$ in the expansion of $(z)_{n}$ are either zero or positive. Thus, A.2 implies

$$
(-1)^{n-v} \mathbf{S}^{(1)}(n, v)=\left|\mathbf{S}^{(1)}(n, v)\right| .
$$


The Stirling numbers $\mathbf{S}^{(2)}(n, v)$ of the second kind are usually defined as follows (see for example [41, Eq. (14) on p. 58]):

$$
z^{n}=\sum_{v=0}^{n} \mathbf{S}^{(2)}(n, v)(z-v+1)_{v}, \quad n \in \mathbb{N}_{0} .
$$

The Stirling numbers of the first and second kind correspond to triangular matrices that transform the polynomial sequences $\left\{(z-n+1)_{n}\right\}_{n=0}^{\infty}$ and $\left\{z^{n}\right\}_{n=0}^{\infty}$ into each other. Since these transformations must be invertible, orthogonality relationships exist.

If we replace in A.1 the powers on the right-hand side by Pochhammer symbols according to A.4, we obtain:

$$
(z-n+1)_{n}=\sum_{v=0}^{n} \mathbf{S}^{(1)}(n, v) \sum_{k=0}^{v} \mathbf{S}^{(2)}(v, k)(z-k+1)_{k}, \quad n \in \mathbb{N}_{0} .
$$

By interchanging the order of the summations summations, this expression can be rewritten as follows:

$$
(z-n+1)_{n}=\sum_{k=0}^{n}(z-k+1)_{k} \sum_{v=k}^{n} \mathbf{S}^{(1)}(n, v) \mathbf{S}^{(2)}(v, k), \quad n \in \mathbb{N}_{0} .
$$

Thus, we obtain the following well-known orthogonality relationship:

$$
\sum_{v=k}^{n} \mathbf{S}^{(1)}(n, v) \mathbf{S}^{(2)}(v, k)=\delta_{n k}, \quad k, n \in \mathbb{N}_{0}
$$

By replacing in the Pochhammer symbols on the right-hand side of A.4 by powers according to A.1), we obtain the following alternative orthogonality relationship:

$$
\sum_{v=k}^{n} \mathbf{S}^{(2)}(n, v) \mathbf{S}^{(1)}(v, k)=\delta_{n k}, \quad k, n \in \mathbb{N}_{0}
$$

The Stirling numbers $\mathbf{S}^{(2)}(n, v)$ of the second kind possess the following infinite generating function (see for example [41, Eq. (16) on p. 58]):

$$
\frac{1}{(1-t)(1-2 t) \ldots(1-k t)}=\sum_{\kappa=0}^{\infty} \mathbf{S}^{(2)}(k+\kappa, k) t^{\kappa}, \quad k \in \mathbb{N}, \quad|t|<1 / k .
$$

The substitution $t=1 / z$ yields:

$$
\frac{1}{(z-k)_{k+1}}=\sum_{\kappa=0}^{\infty} \mathbf{S}^{(2)}(k+\kappa, k) z^{-k-\kappa-1}, \quad|z|>k .
$$

If we now use $(z-k)_{k+1}=(-1)^{k+1}(-z)_{k+1}$ and replace $z$ by $-z$, we obtain the following inverse power series expansion of an inverse Pochhammer symbol [31, Eq. (9) on p. 68]:

$$
\frac{1}{(z)_{k+1}}=\sum_{\kappa=0}^{\infty} \frac{(-1)^{\kappa} \mathbf{S}^{(2)}(k+\kappa, k)}{z^{k+\kappa+1}}, \quad k \in \mathbb{N}_{0}, \quad|z|>k
$$

A convenient starting point for the derivation of a factorial series for an inverse power $1 / z^{k+1}$ is the following factorial series [31, Eq. (3) on p. 77]:

$$
\frac{1}{z-w}=\sum_{n=0}^{\infty} \frac{(w)_{n}}{(z)_{n+1}}, \quad \Re(z-w)>0 .
$$

Next, we apply $\mathrm{d}^{k}[1 /(z-w)] / \mathrm{d} w^{k}=k ! /(z-w)^{k+1}$ with $k \in \mathbb{N}_{0}$ to A.12), which yields:

$$
\frac{k !}{(z-w)^{k+1}}=\frac{\mathrm{d}^{k}}{\mathrm{~d} w^{k}} \sum_{n=0}^{\infty} \frac{(w)_{n}}{(z)_{n+1}} .
$$


The Pochhammer symbol $(w)_{n}$ can be expanded with the help of A.1). Then, the differentiations can be done in closed form and we obtain - after setting $w=0$ - the following factorial series for an inverse power [31, Eq. (6) on p. 78]:

$$
\frac{1}{z^{k+1}}=\sum_{\kappa=0}^{\infty} \frac{(-1)^{\kappa} \mathbf{S}^{(1)}(k+\kappa, k)}{(z)_{k+\kappa+1}}, \quad k \in \mathbb{N}_{0} .
$$

\section{Appendix B. Sequence inversion by triangular orthogonal matrices}

Let us assume that we have two matrices $\mathbf{A}=\left\{A_{m n}\right\}_{m, n \geq 0}$ and $\mathbf{B}=\left\{B_{m n}\right\}_{m, n \geq 0}$, which are triangular,

$$
A_{m n}=B_{m n}=0, \quad n>m
$$

and which satisfy the orthogonality relationships

$$
\begin{aligned}
& \sum_{r=k}^{n} B_{n r} A_{r k}=\delta_{n k}, \\
& \sum_{r=k}^{n} A_{n r} B_{r k}=\delta_{n k} .
\end{aligned}
$$

The orthogonality relationships A.7 and A.8 involving the Stirling numbers of the first and second kind are an example of such a pair of orthogonality relationships.

We also assume that there are two sequences $\left\{x_{n}\right\}_{n=0}^{\infty}$ and $\left\{y_{n}\right\}_{n=0}^{\infty}$ whose elements are connected by finite linear combinations

$$
y_{r}=\sum_{k=0}^{r} A_{r k} x_{k}, \quad r \in \mathbb{N}_{0},
$$

and two sequences $\left\{u_{n}\right\}_{n=0}^{\infty}$ and $\left\{w_{n}\right\}_{n=0}^{\infty}$, whose elements are connected by infinite series expansions

$$
u_{r}=\sum_{n=r}^{\infty} A_{n r} w_{n}, \quad r \in \mathbb{N}_{0} .
$$

In the book of Charalambides [18, Example 8.2 on pp. $284-285]$ it is shown that the finite linear combination (B.4) then possesses the inverse relation

$$
x_{n}=\sum_{k=0}^{n} B_{n k} y_{k}, \quad n \in \mathbb{N}_{0}
$$

and that the infinite series expansion (B.5) then possesses the inverse relation

$$
w_{k}=\sum_{r=k}^{\infty} B_{r k} u_{r}, \quad k \in \mathbb{N}_{0} .
$$

Obviously, the two finite linear combinations (B.4) and (B.6) generalize the finite generating functions (A.1) and (A.4) of the Stirling numbers, and the two infinite series expansions (B.5) and (B.7) generalize the series expansions (A.11) and (A.14) connecting inverse powers and inverse Pochhammer symbols.

Further generalizations are possible. Let us now consider the following, in general formal infinite series expansion:

$$
F=\sum_{n=0}^{\infty} \eta_{n} y_{n}
$$

With the help of $\left(\underline{B .4}\right.$, the elements of the sequence $\left\{y_{n}\right\}_{n=0}^{\infty}$ can be replaced by the elements of the sequence $\left\{x_{n}\right\}_{n=0}^{\infty}$ :

$$
F=\sum_{n=0}^{\infty} \eta_{n} \sum_{k=0}^{n} A_{n k} x_{k}
$$


The order of the summations of this expansion can be rearranged:

$$
\begin{aligned}
F= & x_{0}\left[\eta_{0} A_{00}+\eta_{1} A_{10}+\eta_{2} A_{20}+\ldots\right]+x_{1}\left[\eta_{1} A_{11}+\eta_{2} A_{21}+\eta_{3} A_{31}+\ldots\right] \\
& +x_{2}\left[\eta_{2} A_{22}+\eta_{3} A_{32}+\eta_{4} A_{42}+\ldots\right]+x_{3}\left[\eta_{3} A_{33}+\eta_{4} A_{43}+\eta_{5} A_{53}+\ldots\right]+\ldots \\
= & \sum_{n=0}^{\infty} x_{n} \sum_{k=n}^{\infty} A_{k n} \eta_{k} .
\end{aligned}
$$

Thus, the substitution $\left\{y_{n}\right\}_{n=0}^{\infty} \rightarrow\left\{x_{n}\right\}_{n=0}^{\infty}$ in $\mathrm{B} .8$ produces an alternative expansion for $F$ having the same general structure:

$$
F=\sum_{n=0}^{\infty} \xi_{n} x_{n}, \quad \xi_{n}=\sum_{k=n}^{\infty} A_{k n} \eta_{k}=\sum_{v=0}^{\infty} A_{n+v, n} \eta_{n+v} .
$$

Unknowingly, I had used transformation formulas for expansion coefficients of the kind of (B.12) already in [54] when I studied the transformation of Laguerre series to power series.

Next, we consider the following, in general formal infinite series expansion:

$$
G=\sum_{n=0}^{\infty} v_{n} u_{n}
$$

With the help of $(\mathrm{B} .5)$, the elements of $\left\{u_{n}\right\}_{n=0}^{\infty}$ can be replaced by the elements of $\left\{w_{n}\right\}_{n=0}^{\infty}$ :

$$
G=\sum_{n=0}^{\infty} v_{n} \sum_{k=n}^{\infty} A_{k n} w_{k} .
$$

The order of the summations of this expansion can be rearranged:

$$
\begin{aligned}
G & =w_{0} v_{n} A_{00}+w_{1}\left[A_{10} v_{0}+A_{11} v_{1}\right]+w_{2}\left[A_{20} v_{0}+A_{21} v_{1}+A_{22} v_{2}\right]+\ldots \\
& =\sum_{n=0}^{\infty} w_{n} \sum_{k=0}^{n} A_{n k} v_{k} .
\end{aligned}
$$

Thus, the substitution $\left\{u_{n}\right\}_{n=0}^{\infty} \rightarrow\left\{w_{n}\right\}_{n=0}^{\infty}$ in B.13 produces an alternative expansion of the same general structure:

$$
G=\sum_{n=0}^{\infty} \omega_{n} w_{n}, \quad \omega_{n}=\sum_{k=0}^{n} A_{n k} v_{k} .
$$

These relationships for $G$ obviously generalize the transformation formulas (4.1) and (4.2), which transform factorial series and inverse power series into each other.

Let me emphasize once more that all operations considered in this Section are purely formal. Accordingly, we cannot tacitly assume that the transformation formulas necessarily lead to convergent expansions. This has to be checked explicitly in each case.

This Appendix was inspired by the orthogonality relationships A.7 and A.8 involving the Stirling numbers of the first and second kind. But many other mathematical objects possess similar features. Recently, I had studied the transformation of Laguerre expansions to power series expansions [54]. As is well known, generalized Laguerre polynomials and powers are connected by finite sums of the type of (B.4) and (B.6) which can be inverted. Accordingly, these transformation formulas possess certain orthogonality properties when written in matrix form. Moreover, the coefficients of Laguerre series and of power series are connected by infinite series expansions of the type of (B.12). I only understood the wider significance of these features after [54] was published when I studied the book by Charalambides [18] more carefully.

[1] M. Abramowitz, I. A. Stegun (eds.), Handbook of Mathematical Functions, National Bureau of Standards, Washington, D. C., 1972.

[2] G. A. Baker, Jr., P. Graves-Morris, Padé Approximants, 2nd ed., Cambridge U. P., Cambridge, 1996.

[3] C. M. Bender, S. A. Orszag, Advanced Mathematical Methods for Scientists and Engineers, McGraw-Hill, New York, 1978.

[4] C. M. Bender, T. T. Wu, Anharmonic oscillator, Phys. Rev. 184 (1969) 1231 - 1260.

[5] C. M. Bender, T. T. Wu, Large-order behavior of perturbation theory, Phys. Rev. Lett. 27 (1971) 461 - 465. 
[6] E. Borel, Leçons sur les Séries Divergentes, 2nd ed., Éditions Jacques Gabay, Paris, 1988. Originally published by Gautier-Villars, Paris, 1928. English translation by C. L. Critchfield and A. Vakar, Lectures on Divergent Series, Translation LA-6140-TR, Los Alamos Scientific Laboratory, Los Alamos, 1975.

[7] R. Borghi, Evaluation of diffraction catastrophes by using Weniger transformation, Opt. Lett. 32 (2007) 226 - 228.

[8] R. Borghi, Joint use of the Weniger transformation and hyperasymptotics for accurate asymptotic evaluations of a class of saddle-point integrals, Phys. Rev. E 78 (2008) 026703-1 - 026703-11.

[9] R. Borghi, On the numerical evaluation of cuspoid diffraction catastrophes, J. Opt. Soc. Amer. A 25 (2008) $1682-1690$.

[10] R. Borghi, Summing Pauli asymptotic series to solve the wedge problem, J. Opt. Soc. Amer. A 25 (2008) $211-218$.

[11] R. Borghi, Joint use of the Weniger transformation and hyperasymptotics for accurate asymptotic evaluations of a class of saddle-point integrals. II. Higher-order transformations, Phys. Rev. E 80 (2009) 016704-1 - 016704-15.

[12] R. Borghi, M. Alonso, Efficient evaluation of far-field asymptotic series, in: ICTON '07: 9th International Conference on Transparent Optical Networks, vol. 3, IEEE Xplore, Piscataway, NJ, 2007.

[13] R. Borghi, M. Santarsiero, Summing Lax series for nonparaxial beam propagation, Opt. Lett. 28 (2003) 774 - 776.

[14] C. Brezinski, A. C. Matos, A derivation of extrapolation algorithms based on error estimates, J. Comput. Appl. Math. 66 (1996) 5 - 26.

[15] C. Brezinski, M. Redivo Zaglia, A general extrapolation procedure revisited, Adv. Comput. Math. 2 (1994) 461 - 477.

[16] C. Brezinski, M. Redivo Zaglia, On the kernel of sequence transformations, Appl. Numer. Math. 16 (1994) 239 - 244.

[17] E. Caliceti, M. Meyer-Hermann, P. Ribeca, A. Surzhykov, U. D. Jentschura, From useful algorithms for slowly convergent series to physical predictions based on divergent perturbative expansions, Phys. Rep. 446 (2007) $1-96$

[18] C. A. Charalambides, Enumerative Combinatorics, Chapman \& Hall, Boca Raton, 2002.

[19] J. Č́žžek, J. Zamastil, L. Skála, New summation technique for rapidly divergent perturbation series. Hydrogen atom in magnetic field, J. Math. Phys. 44 (2003) $962-968$.

[20] E. Delabaere, J.-M. Rasoamanana, Sommation effective d'une somme Borel par séries de factorielles, Annal. l'Inst. Fourier 57 (2007) 421 456.

[21] S. Graffi, V. Grecchi, B. Simon, Borel summability: Application to the anharmonic oscillator, Phys. Lett. B 32 (1970) $631-634$.

[22] K. Knopp, Theorie und Anwendung der unendlichen Reihen, Springer-Verlag, Berlin, 1964.

[23] E. Landau, Über die Grundlagen der Theorie der Fakultätenreihen, Sitzungsb. Königl. Bay. Akad. Wissensch. München, math.-phys. Kl. 36 (1906) $151-218$

[24] J. C. Le Guillou, J. Zinn-Justin (eds.), Large-Order Behaviour of Perturbation Theory, North-Holland, Amsterdam, 1990.

[25] D. Levin, Development of non-linear transformations for improving convergence of sequences, Int. J. Comput. Math. B 3 (1973) 371 - 388.

[26] J. Li, W. Zang, J. Tian, Simulation of Gaussian laser beams and electron dynamics by Weniger transformation method, Opt. Expr. 17 (2009) $4959-4969$.

[27] J.-X. Li, W. Zang, Y.-D. Li, J. Tian, Acceleration of electrons by a tightly focused intense laser beam, Opt. Expr. 17 (2009) $11850-11859$.

[28] B. Malgrange, Sommation des sries divergentes, Expo. Math. 13 (1995) $163-222$.

[29] L. M. Milne-Thomson, The Calculus of Finite Differences, Chelsea, New York, 1981. Originally published by Macmillan, London, 1933.

[30] F. Nevanlinna, Zur Theorie asymptotischer Potenzreihen, Ann. Acad. Sci. Fenn. Ser. A 12 (1919) $1-81$.

[31] N. Nielsen, Die Gammafunktion, Chelsea, New York, 1965. Originally published by Teubner, Leipzig and Berlin, 1906.

[32] N.-E. Nörlund, Leçons sur les Séries d'Interpolation, Gautier-Villars, Paris, 1926.

[33] N.-E. Nörlund, Leçons sur les Équations Linéaires aux Différences Finies, Gautier-Villars, Paris, 1929.

[34] N. E. Nörlund, Vorlesungen über Differenzenrechnung, Chelsea, New York, 1954. Originally published by Springer-Verlag, Berlin, 1924.

[35] A. B. Olde Daalhuis, Inverse factorial-series solutions of difference equations, Proc. Edinb. Math. Soc. 47 (2004) 421 - 448.

[36] J. P. Ramis, J. Thomann, Some comments about the numerical utilization of factorial series, in: J. Della Dora, J. Demongeot, B. Lacolle (eds.), NumericaL Methods in the Study of Critical Phenomena, Springer-Verlag, Berlin, 1981, pp. $12-25$.

[37] B. Simon, Coupling constant analyticity for the anharmonic oscillator, Ann. Phys. (NY) 58 (1970) $76-136$.

[38] B. Simon, Fifty years of eigenvalue perturbation theory, Bull. Amer. Math. Soc. 24 (1991) $303-319$.

[39] D. A. Smith, W. F. Ford, Acceleration of linear and logarithmic convergence, SIAM J. Numer. Anal. 16 (1979) $223-240$.

[40] D. A. Smith, W. F. Ford, Numerical comparisons of nonlinear convergence accelerators, Math. Comput. 38 (1982) $481-499$.

[41] H. M. Srivastava, J. Choi, Series Associated with the Zeta and Related Functions, Kluwer, Dordrecht, 2001.

[42] J. Thomann, Resommation des series formelles, Numer. Math. 58 (1990) $503-535$.

[43] J. Thomann, Procédés formels et numériques de sommation de séries d'équations differentielles, Expo. Math. 13 (1995) 223 - 246.

[44] I. Tweddle, James Stirling's Methodus Differentialis: An Annotated Translation of Stirling's Text, Springer-Verlag, London, 2003.

[45] G. Watson, The transformation of an asymptotic series into a convergent series of inverse factorials, Rend. Circ. Mat. Palermo 34 (1912) 41 -88 .

[46] E. J. Weniger, Nonlinear sequence transformations for the acceleration of convergence and the summation of divergent series, Comput. Phys. Rep. 10 (1989) 189 - 371, Los Alamos Preprint math-ph/0306302 (http://arXiv.org).

[47] E. J. Weniger, On the summation of some divergent hypergeometric series and related perturbation expansions, J. Comput. Appl. Math. 32 (1990) $291-300$.

[48] E. J. Weniger, Interpolation between sequence transformations, Numer. Algor. 3 (1992) 477 - 486.

[49] E. J. Weniger, Verallgemeinerte Summationsprozesse als numerische Hilfsmittel für quantenmechanische und quantenchemische Rechnungen, Habilitation thesis, Fachbereich Chemie und Pharmazie, Universität Regensburg, Los Alamos Preprint math-ph/0306048 (http://arXiv.org) (1994).

[50] E. J. Weniger, A convergent renormalized strong coupling perturbation expansion for the ground state energy of the quartic, sextic, and octic anharmonic oscillator, Ann. Phys. (NY) 246 (1996) 133 - 165.

[51] E. J. Weniger, Construction of the strong coupling expansion for the ground state energy of the quartic, sextic and octic anharmonic oscillator via a renormalized strong coupling expansion, Phys. Rev. Lett. 77 (1996) $2859-2862$.

[52] E. J. Weniger, Mathematical properties of a new Levin-type sequence transformation introduced by Čížek, Zamastil, and Skála. I. Algebraic 
theory, J. Math. Phys. 45 (2004) 1209 - 1246.

[53] E. J. Weniger, Asymptotic approximations to truncation errors of series representations for special functions, in: A. Iske, J. Levesley (eds.), Algorithms for Approximation, Springer-Verlag, Berlin, 2007.

[54] E. J. Weniger, On the analyticity of Laguerre series, J. Phys. A 41 (2008) 425207-1 - 425207-43.

[55] E. J. Weniger, J. Č́žzek, F. Vinette, The summation of the ordinary and renormalized perturbation series for the ground state energy of the quartic, sextic, and octic anharmonic oscillators using nonlinear sequence transformations, J. Math. Phys. 34 (1993) 571 - 609.

[56] E. J. Weniger, E. O. Steinborn, Nonlinear sequence transformations for the efficient evaluation of auxiliary functions for GTO molecular integrals, in: M. Defranceschi, J. Delhalle (eds.), Numerical Determination of the Electronic Structure of Atoms, Diatomic and Polyatomic Molecules, Kluwer, Dordrecht, 1989, pp. 341 - 346. 